Musées, Patrimoine et Culture scientifiques et techniques

$153 \mid 2014$

mai-juin 2014

\title{
Le traitement patrimonial de fonds scientifiques
}

Joël Clary

URL : http://journals.openedition.org/ocim/1397

DOI : 10.4000/ocim.1397

ISSN : 2108-646X

Éditeur

OCIM

Édition imprimée

Date de publication : 25 juin 2014

ISSN : 0994-1908

\section{Référence électronique}

Joël Clary, «Le traitement patrimonial de fonds scientifiques », La Lettre de l'OCIM [En ligne],

153 | 2014, mis en ligne le 25 juin 2016, consulté le 01 mai 2019. URL : http://journals.openedition.org/ ocim/1397 ; DOl : 10.4000/ocim.1397 


\section{Le traitement patrimonial de fonds scientifiques}

\section{Joël Clary}

Conservateur du patrimoine au musée des Confluences, Lyon

Dans le domaine de l'Histoire naturelle, le matériel d'étude comprend volontiers des produits de fouilles paléontologiques ou de collectes d'échantillons végétaux ou animaux. Il peut s'agir de collectes actuelles ou de fonds de collection anciens ni triés ni inventoriés jusque-là.

Assez régulièrement, des fonds scientifiques sont proposés au musée des Confluences par des familles de collectionneurs entomologistes ou conchyliologues disparus. Les collections qu'ils renferment ont quelquefois été laissées à l’abandon, voire vandalisées. Schématiquement, ces ensembles, que l'on peut qualifier de

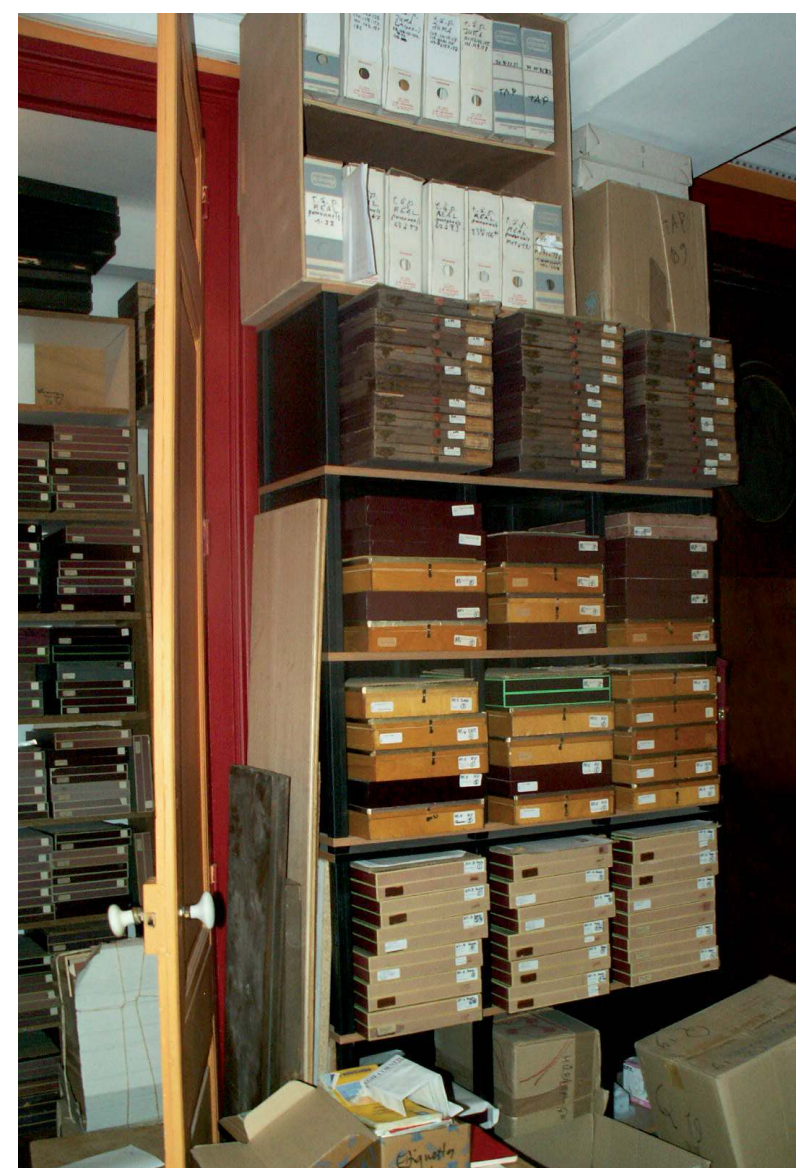

Le travail de repérage documentaire in situ est important pour une collection scientifique proposée en donation

(Collection P. Réal, musée des Confluences). ๑) Département du Rhône/musée des Confluences/Joël Clary complexes, comprennent des lots d'objets - avec différents modes de préparation des spécimens - parfois incomplets et plus ou moins bien classés, et toute la documentation afférente. Leur transfert au musée doit généralement être mené dans l'urgence et la formalisation d'un dépôt conservatoire s'avère alors nécessaire. Depuis de nombreuses années déjà, nous avons choisi d'appliquer les modalités de traitement du matériel d'étude à ce type d'acquisition. En revanche, les collections ne nécessitant qu'une intervention de tri minimale ont pu rejoindre rapidement le fonds patrimonial de l'institution.

Sur place, un travail préparatoire a pour but d'établir un premier état des lieux du fonds, d'en cerner les principaux éléments et d'en établir une brève description quantitative et qualitative. Il convient naturellement d'en garder la mémoire sous forme de photographies ou de scans. Parallèlement, il est indispensable de rassembler un maximum d'informations sur l'auteur, la nature et la portée de son travail (biographie), ses productions écrites (bibliographie). La consultation de la documentation (notes, répertoires, listes, publications, courriers...) doit permettre de mieux cerner l'identité de la collection, la logique et le mode de classement du matériel, et permettre d'en dégager les grandes composantes. Les échanges avec l'auteur ou sa famille sont importants pour tenter de déchiffrer et de comprendre le fonds, tenter de s'imprégner de l'esprit avec lequel il a été constitué.

Après son transfert au musée, on peut commencer à hiérarchiser le matériel selon son intérêt scientifique - avec une attention particulière pour les parties en mauvais état - repérer les lots mis de côté par l'auteur en raison d'un intérêt particulier, localiser ou tout du moins suspecter l'existence de biens remarquables comme les types, les spécimens publiés ou étudiés. La collection peut être formée de plusieurs ensembles - nous avons eu ainsi à traiter un fonds de plus de 1000 cartons d'insectes comprenant 6 ensembles distincts et parfois renfermer du matériel emprunté à d'autres spécialistes ou à des musées.

À ce stade, il s'avère souvent indispensable de compléter l'expertise de la collection en faisant appel à une aide extérieure. Celle-ci peut se concrétiser par un 


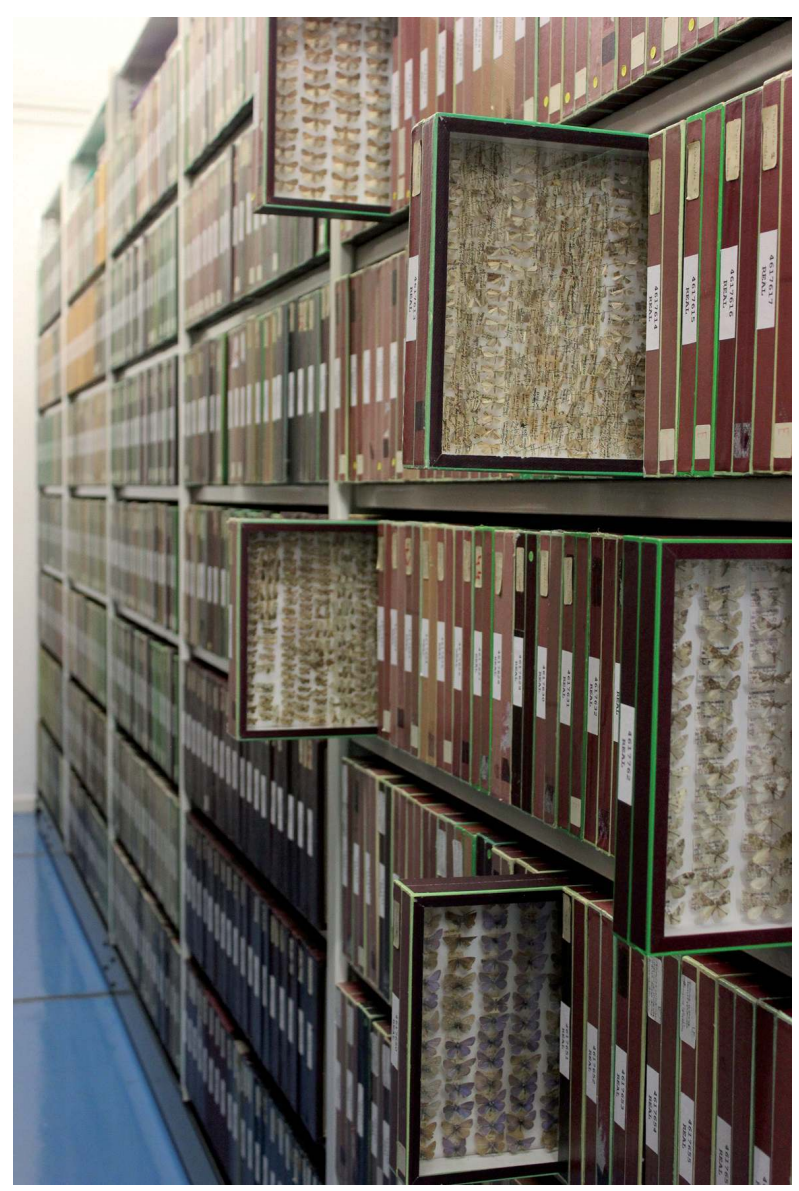

Cinq années auront été nécessaires au traitement de la collection et à son rangement définitif en réserve (Collection P. Réal, musée des Confluences). ○ Département du Rhône/musée des Confluences/Joël Clary

échange d’informations mais nécessite généralement une étude du matériel sur place. Sur le plan scientifique, il convient de vérifier la présence potentielle de «types »; au niveau historique, on s'intéresse à la spécificité et à l'originalité des pratiques du collectionneur, ce qui conduit parfois à conserver des éléments témoins : nous avons par exemple fait le choix de conserver une caisse d'expédition de matériel très originale, quelques instruments de préparation et d'observation ou encore des boîtes de stockage de coquilles singulières. Ces données scientifiques et historiques sont alors croisées avec l'état et le statut du matériel (propriété), ce qui permet d'en achever le tri et de distinguer les éléments entrant au patrimoine. Le reclassement final de la collection, qui doit respecter l'ordre originel ou garder l'esprit du classement, est une opération relativement longue - entre 3 et 7 ans pour les cas traités. Elle peut s'avérer fastidieuse dans le cas d'une collection complexe ou très endommagée.
La proportion de matériel patrimonial soumis à la commission scientifique régionale varie selon la nature, la qualité et l'état des ensembles que nous avons eu à traiter, de 50 à $95 \%$. Une partie rejoint le fonds de matériel pédagogique, conservé ou cédé à une autre institution, une association naturaliste par exemple. La proportion de ce matériel a pu atteindre $50 \%$ pour un ensemble entomologique comprenant beaucoup de matériel qui n'était pas préparé. Le matériel restitué revient pour une faible part à la famille qui souhaite conserver un témoignage. On arrive généralement à la convaincre de ne pas conserver le matériel scientifique pouvant intéresser le musée. Il n'est pas rare de retrouver du matériel emprunté à d'autres institutions ; il doit être restitué en prenant la précaution préalable d'obtenir la confirmation de ces prêts. La proportion de matériel détruit est également variable. Dans notre cas, elle a pu avoisiner les 30 \% pour une collection très endommagée et $80 \%$ pour une autre peu spécialisée.

Le propos est bien ici de pérenniser des ensembles scientifiques importants en respectant l'intention première de leurs auteurs. Face à une volonté de patrimonialisation rapide mais devant l'ampleur de la tâche, grande est la tentation d'en repousser l'échéance et de conserver ces fonds en l'état et... pour un certain temps. Aussi, l'opportunité de traiter ces ensembles complexes sous la forme de matériel d'étude procuret-elle l'avantage d'une démarche scientifique et patrimoniale sur le court terme, garantissant une perte minimale d'informations sur la collection. Cependant, une telle démarche nécessite une réelle motivation, un investissement en temps et en moyens ainsi qu'une mobilisation d'intervenants extérieurs dont la mise en action n'est pas toujours aisée.

L'auteur remercie Cédric Audibert du musée des Confluences pour ses commentaires. 